\title{
A Novel Endoscopic Mouthpiece for COVID-19 Prevention
}

\author{
Takuto Hikichi', Nakamura Jun ${ }^{1}$, Koichi Hamada ${ }^{2}$, and Daiki Nemoto ${ }^{3}$ \\ ${ }^{1}$ Department of Endoscopy, Fukushima Medical University Hospital, Fukushima, Fukushima, ${ }^{2}$ Department of Gastroenterology, \\ Southern Tohoku General Hospital, Koriyama, Fukushima, ${ }^{3}$ Department of Coloproctology, Fukushima Medical University Aizu Medical \\ Center, Aizuwakamatsu, Fukushima, Japan
}

During the present coronavirus disease 2019 (COVID-19) pandemic, gastrointestinal endoscopy is regarded as a risky procedure because virus transmission can occur via an airborne route. To reduce the spread of aerosolized droplets, several devices have been developed. ${ }^{1-7}$ This novel mouthpiece consists of a conventional mouthpiece made of polypropylene and a hat-shaped aerosol droplet guard, which are connected together. (Fig. 1A and B). The aerosol droplet guard, which is made of a thermoplastic elastomer, has a width of $90 \mathrm{~mm}$ and a height of $50 \mathrm{~mm}$, and can prevent aerosolized droplets from being ejected from the mouth (Fig. 1C). The center of the aerosol droplet guard has four slits that are $6 \mathrm{~mm}$ long and are oriented to allow the scope to pass through them. (Fig. 1D), while also acting as valves to prevent aerosolized droplet diffusion.
The effectiveness of the new mouthpiece in preventing aerosolized droplet diffusion was compared to that of a conventional mouthpiece. As an experimental model, simulated saliva was sprayed from the mouth of a mannequin using a cough machine. The simulated saliva was prepared by adding $76 \mathrm{~g}$ of glycerin to $1 \mathrm{~L}$ of water. ${ }^{8}$ With the endoscope inserted into the mouthpiece, saliva was sprayed in the following conditions: duration, $0.2 \mathrm{sec}$; velocity, $10.7 \mathrm{~m} / \mathrm{sec}$; volume, 75.4 $\mathrm{mL}$; and pressure, $0.08 \mathrm{MPa}^{.}{ }^{8}$ Three technical repeats were completed, and the diffusion of the aerosolized droplets was photographed by a camera in a darkened room with a Polarion light. Although there was significant aerosolized droplet diffusion when using the conventional mouthpiece (Fig. 2A), it was only minimally detectable with the new mouthpiece (Fig. 2B).
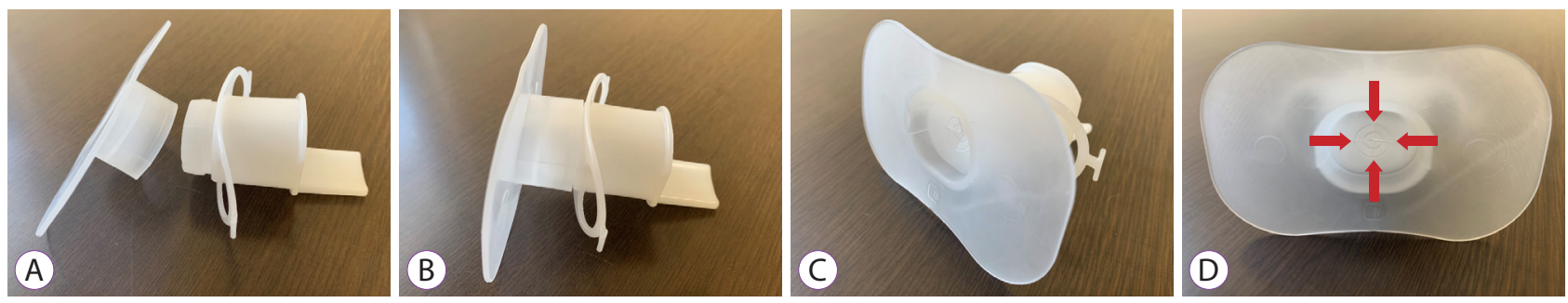

Fig. 1. Appearance of the new mouthpiece. (A, B) Side view. The mouthpiece is connected to a hat-shaped aerosol droplet guard. (C, D) Front view. The guard prevents aerosolized droplets from exiting the mouth when the patient coughs. The red arrow shows the slit that the scope is passed through.

\footnotetext{
Received: May 31, $2021 \quad$ Revised: June 1, 2021

Accepted: June 2, 2021

Correspondence: Takuto Hikichi

Department of Endoscopy, Fukushima Medical University Hospital, Fukushima-city, Fukushima 960-1295, Japan

Tel: +81-24-547-1583, Fax: +81-24-547-1586, E-mail: takuto@fmu.ac.jp

ORCID: https://orcid.org/0000-0002-9815-1557
}

(cc) This is an Open Access article distributed under the terms of the Creative Commons Attribution Non-Commercial License (http://creativecommons.org/licenses/by$\mathrm{nc} / 3.0$ ) which permits unrestricted non-commercial use, distribution, and reproduction in any medium, provided the original work is properly cited. 
The aerosol droplet guard not only prevents diffusion in the area from where the scope is inserted, but also blocks diffusion from the gap between the mouthpiece and the mouth, which is a distinct feature not found in conventional mouthpieces. As the new mouthpiece has not yet been applied clinically, its effect on respiratory and circulatory dynamics during endos- copy is still unknown. However, when used in a virtual clinical setting, there was no apparent breathing discomfort (Fig. 3A and $\mathrm{B}$ ).

In conclusion, this new mouthpiece can protect clinicians from infection when carrying out endoscopic procedures, especially in the wake of the COVID-19 pandemic.
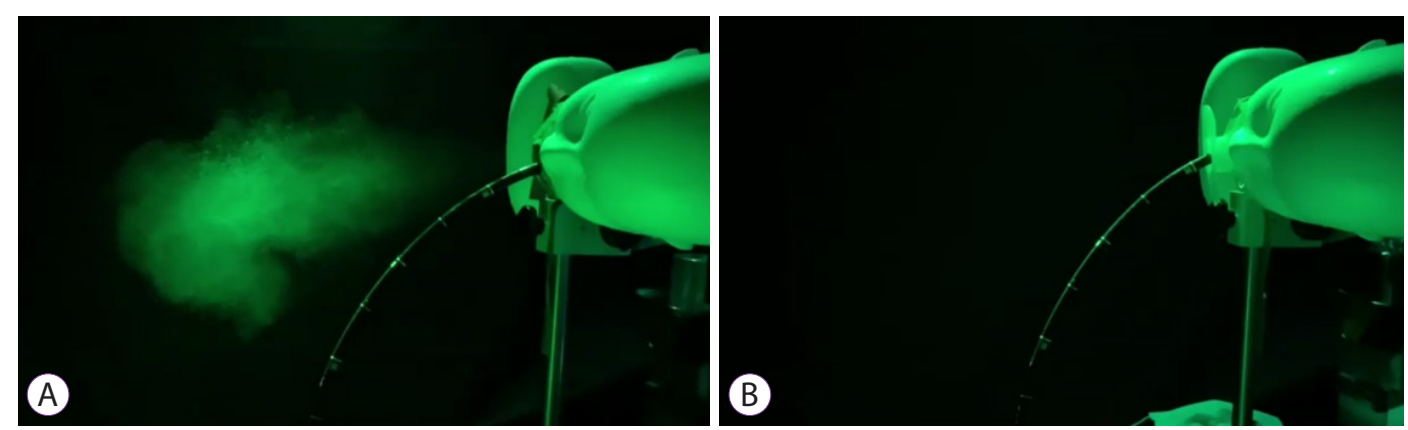

Fig. 2. A comparison of the spread of aerosolized droplets during coughing. (A) Significant droplet diffusion was observed when using a conventional mouthpiece. (B) Significant reduction in the amount of diffused droplets when using the new mouthpiece.
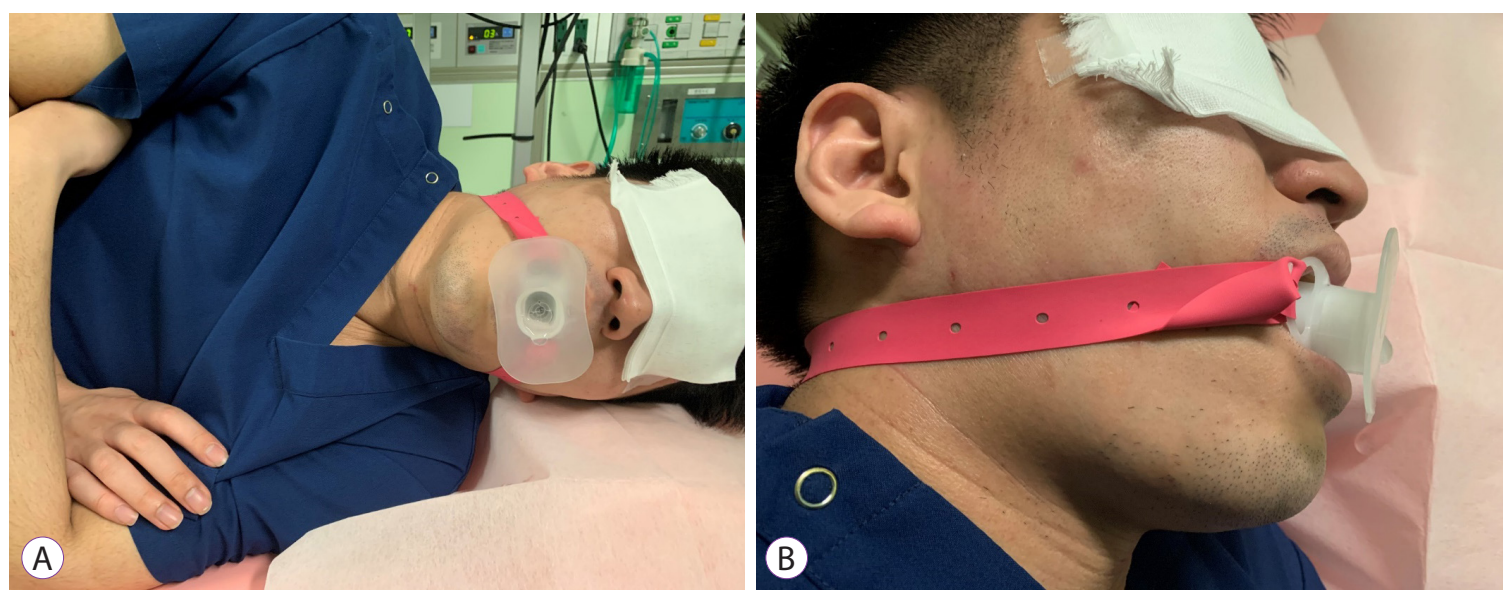

Fig. 3. The virtual clinical application of the mouthpiece. (A) Front view. The hat-shaped aerosol droplet guard is larger than a person's mouth. (B) The aerosol-droplet guard is located $16.5 \mathrm{~mm}$ from the mouth and therefore does not affect respiration.

Conflicts of Interest

The authors have no potential conflicts of interest.

\section{Funding}

None.

\section{Acknowledgments}

We wish to express our deepest appreciation to Mr. Ichiro Sato and Mr. Taiki Ogawa at KSM Co., Ltd. for creating the mouthpiece; to Mr. Shoichi
Uratani, Ms. Rieko Takahashi, and Mr. Kenichi Tabata at the Advanced Technology Center, corporate R\&D headquarters, Konica Minolta, Inc. for their basic experimental support; to Mr. Tsuyoshi Ishibashi and Ms. Emiko Hayashi at the Medical Industry Cluster Promotion Unit, Fukushima Prefectural Government; to Mr. Keita Suzuki at the Fukushima Medical Device Industry Promotion Agency for their advice on this study; to Dr. Ryoichiro Kobashi at the Department of Endoscopy, Fukushima Medical University Hospital; and to Dr. Hiroshi Shimizu and Dr. Naoto Abe at the Department of Gastroenterology, Fukushima Medical University for their basic experimental support and advice on this study. 


\section{Author Contributions}

Conceptualization: Takuto Hikichi

Investigation: $\mathrm{TH}$

Methodology: TH, Jun Nakamura, Koichi Hamada, Daiki Nemoto

Project administration: TH

Resources: TH

Supervision: $\mathrm{TH}$

Validation: $\mathrm{TH}$

Visualization: $\mathrm{TH}$

Writing-original draft: $\mathrm{TH}$

Writing-review\&editing: JN, KH, DN

\section{ORCID}

Takuto Hikichi

Jun Nakamura

Koichi Hamada

Daiki Nemoto

\section{REFERENCES}

1. Sasaki S, Nishikawa J, Sakaida I. Use of a glove-covered mouthpiece during upper endoscopy to prevent COVID-19 transmission. Clin En- dosc 2021;54:289-290.

2. Lazaridis N, Skamnelos A, Murino A, Cahuin RC, Koukias N, Despott EJ. "Double-surgical-mask-with-slit" method: reducing exposure to aerosol generation at upper gastrointestinal endoscopy during the COVID-19 pandemic. Endoscopy 2020;52:928-929.

3. Endo H, Koike T, Masamune A. Novel device for preventing diffusion of aerosol droplets from subjects undergoing esophagogastroduodenoscopy during COVID-19 pandemic. Dig Endosc 2020;32:e140-e141.

4. Maruyama H, Higashimori A, Yamamoto K, et al. Coronavirus disease outbreak: a simple infection prevention measure using a surgical mask during endoscopy. Endoscopy 2020;52:E461-E462.

5. Bojórquez A, Larequi FJZ, Betés MT, Súbtil JC, Muñoz-Navas M. Commercially available endoscopy facemasks to prevent aerosolizing spread of droplets during COVID-19 outbreak. Endosc Int Open 2020;8:E815-E816.

6. Sagami R, Nishikiori H, Sato T, Murakami K. Endoscopic shield: barrier enclosure during the endoscopy to prevent aerosol droplets during the COVID-19 pandemic. VideoGIE 2020;5:445-448.

7. Kobara H, Nishiyama N, Masaki T. Shielding for patients using a single-use vinyl-box under continuous aerosol suction to minimize SARS-CoV-2 transmission during emergency endoscopy. Dig Endosc 2020;32:e114-e115.

8. Ogata M, Ichikawa M, Tsutsumi H, Ariga T, Hori S, Tanabe S. Measurement of cough droplet deposition using the cough machine. J Environ Eng 2018;83:57-64 